\title{
Present state and promises to unravel the structure and kinematics of the Milky Way with the RAVE survey
}

\author{
M. Steinmetz ${ }^{1}$, A. Siebert ${ }^{2}$, T. Zwitter ${ }^{3}$ and the RAVE collaboration \\ ${ }^{1}$ Astrophysikalisches Institut Potsdam, Potsdam, Germany \\ ${ }^{2}$ Observatoire de Strasbourg, Strasbourg, France \\ ${ }^{3}$ University of Ljubljana, Faculty of Mathematics and Physics, Ljubljana, Slovenia
}

\begin{abstract}
The RAdial Velocity Experiment (RAVE) is an ambitious survey to measure the radial velocities, temperatures, surface gravities, metallicities and abundance ratios for up to a million stars using the 1.2-m UK Schmidt Telescope of the Anglo-Australian Observatory (AAO), over the period 2003-2011. The survey represents a big advance in our understanding of our own Milky Way galaxy. The main data product will be a southern hemisphere survey of about a million stars. Their selection is based exclusively on their $I$-band colour, so avoiding any colour-induced bias. RAVE is expected to be the largest spectroscopic survey of the Solar neighbourhood in the coming decade, but with a significant fraction of giant stars reaching out to $10 \mathrm{kpc}$ from the Sun. RAVE offers the first truly representative inventory of stellar radial velocities for all major components of the Galaxy. Here we present the first scientific results of this survey as well as its second data release which doubles the number of previously released radial velocities. For the first time, the release also provides atmospheric parameters for a large fraction of the second year data, making it an unprecedented tool to study the formation of the Milky Way. Plans for further data releases are outlined.
\end{abstract}

Keywords. catalogs, stars: fundamental parameters, surveys, Galaxy: stellar content, Galaxy: kinematics and dynamics

\section{Introduction}

It is now widely accepted that the Milky Way galaxy is a suitable laboratory to study the formation and evolution of galaxies. Despite the fact that the Galaxy is one unique system, understanding its formation holds important keys to study the broader context of disc galaxy formation. Thanks to the past and ongoing large surveys such as Hipparcos, SDSS, 2MASS or DENIS, we have access to data which allow us to refine our knowledge of Galaxy formation. However, with the exception of the SDSS survey, which mainly samples the halo of the Galaxy, the full description of the $6 \mathrm{D}$ phase space, i.e. the combination of the position and velocity spaces, is not available due to the missing radial velocity and/or distance.

With the advent of multi-fiber spectroscopy, combined to the large field of view of Schmidt telescopes, it is now possible to acquire in a reasonable amount of time spectra for a large sample of stars that is representative for the different populations of the Galaxy. Spectroscopy enables us to measure the generally missing radial velocity, which in turn allows us to study the details of Galactic dynamics. Spectroscopy also permits to measure the abundance of chemical elements in a stellar atmosphere which holds important clues on the initial chemical composition and its subsequent metal enrichment. The measurement of the radial velocity and of the chemical abundances as well as the 
derivation of stellar temperature and gravity in order to complement existing catalogues is the main purpose of the RAVE project.

RAVE is using the $6 \mathrm{dF}$ multi-fiber spectroscopic facility at the UK Schmidt telescope of the Anglo-Australian Observatory in Siding Spring, Australia. The 6dF enables us to collect up to 150 spectra in one single pointing, with an average resolution of 7500 in the Calcium triplet region around $8500 \AA$. This medium resolution allows the measurement of accurate radial velocities $\left(\sim 2 \mathrm{~km} \mathrm{~s}^{-1}\right)$ as well as atmospheric parameters $\left(\mathrm{T}_{\text {eff }}, \log g\right.$, $[\mathrm{M} / \mathrm{H}])$ and chemical abundances. In Section 2 we present the first scientific outcomes obtained using the RAVE data, while Section 3 presents the second data release of the RAVE project (DR2) and discusses the current status and prospects of the project.

\section{First Results from the Survey}

In this section we outline some of the recently published results based on RAVE data.

\subsection{The Escape Velocity of the Galaxy}

In Smith et al. (2007) we revisited the local escape speed of our Galaxy by combining a sample of high velocity stars detected by the RAVE survey with previously known ones. Using a maximum likelihood technique, we find $498<v_{e s c}<608 \mathrm{~km} \mathrm{~s}^{-1}$ at the $90 \%$ confidence level, with a median likelihood of $544 \mathrm{~km} \mathrm{~s}^{-1}$. This result demonstrates the presence of a dark halo in the Milky Way, but simultaneously argues for a halo of relatively low circular velocity $\left(v_{c} \approx 140 \mathrm{~km} \mathrm{~s}^{-1}\right)$.

\subsection{Streams (or lack thereof) in the Solar Neighborhood}

In Seabroke et al. (2008) we searched the CORAVEL and RAVE survey for signatures of vertically infalling stellar streams in the Solar vicinity. Using a Kuiper test, we demonstrated that the Solar neighborhood is empty of any vertical streams containing more than a few hundreds of stars. Therefore, we confirm recent simulations that are favoring a model in which the Sagittarius stream is not entering the Solar neighborhood. We also argue against the Virgo overdensity crossing the disc near the Sun.

\subsection{The Vertical Structure of the Galactic Disc}

Combining RAVE data with photometric and astrometric catalogues, in Veltz et al. (2008) we were using G- and K-type stars towards the Galactic poles in order to identify whether there is a kinematic discontinuity between the thin and thick discs. We conclude that such a discontinuity indeed exists, which is a strong constraint on the formation scenario of the thick disc: it is arguing against continuous processes such as scattering by spiral stellar or molecular arms, but favoring violent processes such as the accretion of or violent heating by a satellite.

\subsection{The Tilt of the Velocity Ellipsoid}

Using RAVE red clump giants towards the South Galactic pole, in Siebert et al. (2008) we have measured the inclination of the velocity ellipsoid at $1 \mathrm{kpc}$ below the Galactic plane. The value of the tilt, $7.3 \pm 1.8^{\circ}$, is consistent with either a short scale length for the disc $\left(R_{d} \sim 2 \mathrm{kpc}\right)$ if the halo is oblate, or a long scale length $\left(R_{d} \sim 3 \mathrm{kpc}\right)$ if the halo is prolate. Combined to independent measurements of the minor-to-major axis ratio of the halo, which prefers an almost spherical halo. A scale length of the disc in the range $[2.5-2.7] \mathrm{kpc}$ is preferred. 


\subsection{Diffuse Interstellar Bands}

In Munari et al. (2008) we used spectra of hot stars from the RAVE survey to investigate the properties of 5 diffuse interstellar bands (DIB) in the Ca triplet region. Our findings indicate that the DIB at $8620.4 \AA$ is strongly correlated to reddening and follows the relation $E(B-V)=(2.72 \pm 0.03) \times \mathrm{EW}$, where $\mathrm{EW}$ is the equivalent width of the DIB in $\AA$. This DIB is thus a suitable tracer of general Galactic reddening in stellar spectra. On the other hand the existence of the DIB at $8648 \AA$ is confirmed, but its intensity or equivalent width does not appear to correlate with reddening.

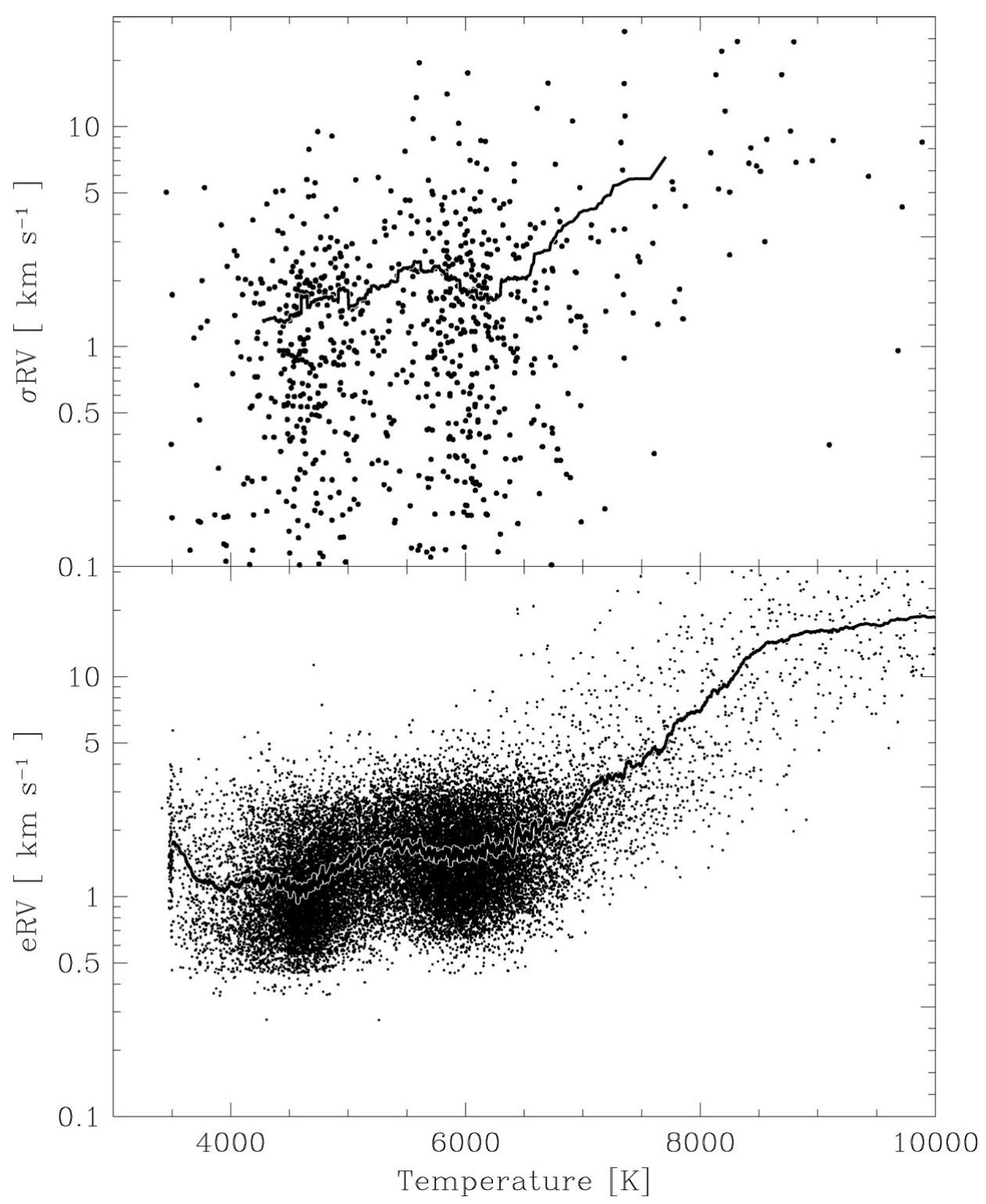

Figure 1. Radial velocity errors for stars in the second RAVE data release as a function of stellar temperature. The error is the internal velocity error (bottom) and the standard deviation of velocities determined from repeated observations of the same object (top). Lines trace a smoothed average dependence, with a boxcar width of 100 points. 


\section{Second data release: Current Status and Ongoing efforts}

\subsection{Second Data Release}

In July 2008, the RAVE collaboration has released the 2nd catalogue of reduced RAVE data (DR2) (Zwitter et al. 2008). DR2 covers an area of $\sim 7200 \mathrm{deg}^{2}$ in the southern hemisphere at galactic latitudes larger than $25^{\circ}$. As for the first data release (DR1) (Steinmetz et al. 2006), the magnitude range is $9 \leqslant I_{I C} \leqslant 12$, where $I_{I C}$ is the $I$ magnitude of input catalogue. It equals the SuperCosmos photographic $I$ magnitude for faint stars $\left(11<I_{I C}<12\right)$, while for bright stars $\left(I_{I C}<11\right)$ it was derived from the Tycho- $2 B_{T}$ and $V_{T}$ magnitudes.

DR2 contains 51,829 radial velocity measurements for 49,327 individual stars, with a typical radial velocity error of 1.3 to $1.7 \mathrm{~km} \mathrm{~s}^{-1}$ for data collected after DR1. Figure 1 shows the dependence of the radial velocity errors on temperature, as determined from RAVE spectra. Radial velocities are marginally better determined for cool giants than for medium temperature main sequence stars. The errors increase for stars of an early spectral type.

DR2 doubles the number of radial velocities previously published in DR1. The RAVE collaboration is continuing its calibration campaign. The comparison to external data shows a standard deviation of $1.3 \mathrm{~km} \mathrm{~s}^{-1}$ for the radial velocities, which is twice better than for the first data release.

In addition to radial velocities, the catalogue contains atmospheric parameters, $\log g$, $[\mathrm{M} / \mathrm{H}]$ and $\mathrm{T}_{\text {eff }}$, for the first time. These parameters were determined based on 22,407 spectra corresponding to 21,121 individual stars. A conservative error estimates for these parameters for the average signal-to-noise ratio of the survey $(\mathrm{S} / \mathrm{N} \sim 40$ ) is $400 \mathrm{~K}$ for the effective temperature, 0.5 dex for the logarithm of the gravity $(\log g)$ and 0.2 dex for the metallicity. These errors depend strongly on S/N. Stars at the extreme ends of the $\mathrm{S} / \mathrm{N}$ range have errors $\sim 2$ times better/worse. The calibration of the RAVE atmospheric

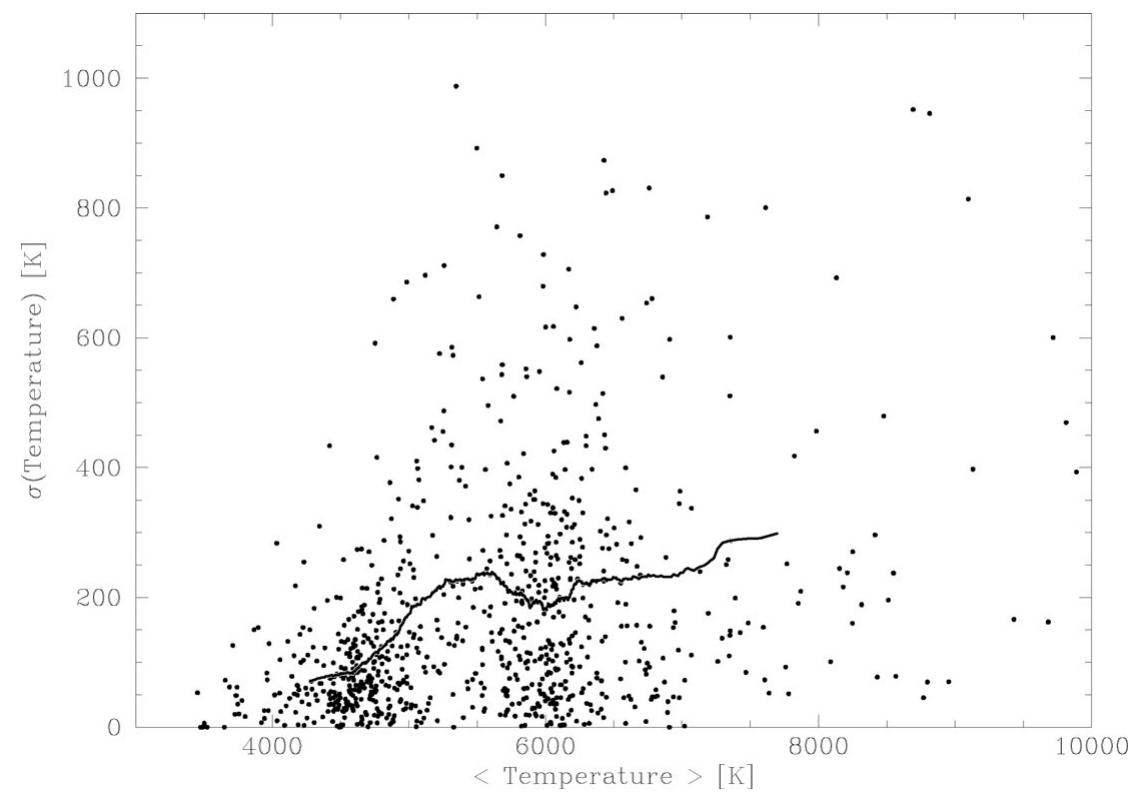

Figure 2. Temperature accuracy as judged from repeated observations of the same object in the second data release. Standard deviation of temperature is given as a function of its average value. The line is a smoothed average using the adjacent 50 cooler and 50 hotter objects. 
parameters is obtained using a devoted validation campaign where high-resolution spectra of standard stars have been taken with various instruments and compared to spectra obtained by RAVE.

Figures 2 to 4 show standard deviations of temperature, gravity, and metallicity as obtained from repeated observations where a certain star was observed more than once by RAVE. The results show that typical errors are only $\lesssim 200 \mathrm{~K}$ in effective temperature, $\sim 0.25$ dex in gravity and $\lesssim 0.15$ dex in metallicity.

The catalogue is available from the RAVE website www.rave-survey.org or at the CDS using the VizieR database.

\subsection{Pilot Survey}

The RAVE pilot survey (data collected between April 2003 and February 2006) is now completed. A data release is scheduled for mid-2009. It contains about 85,000 radial velocity measurements as well as measurements of stellar atmospheric parameters for a large fraction of the sample. The release of the pilot survey will mark a major step forward in the RAVE project, and subsequent data releases will be based on a new input catalogue drawn from the DENIS survey.

\subsection{Current Status}

RAVE currently operates at its full potential, observing is scheduled for 25 nights per lunation. The project entered its main survey phase in March 2006. The main survey relies on a new input catalogue based on DENIS I-band magnitudes. RAVE has so far collected approximately 215,000 spectra in its main phase, which adds to a total of 301,000 spectra for 270,000 stars when combining the two phases of the project. Figure 5 plots the density of spectra per square degree observed before 6th of July 2008. The survey now covers almost the entire Southern hemisphere with the exception of the Galactic plane where only a few test observations have been obtained.

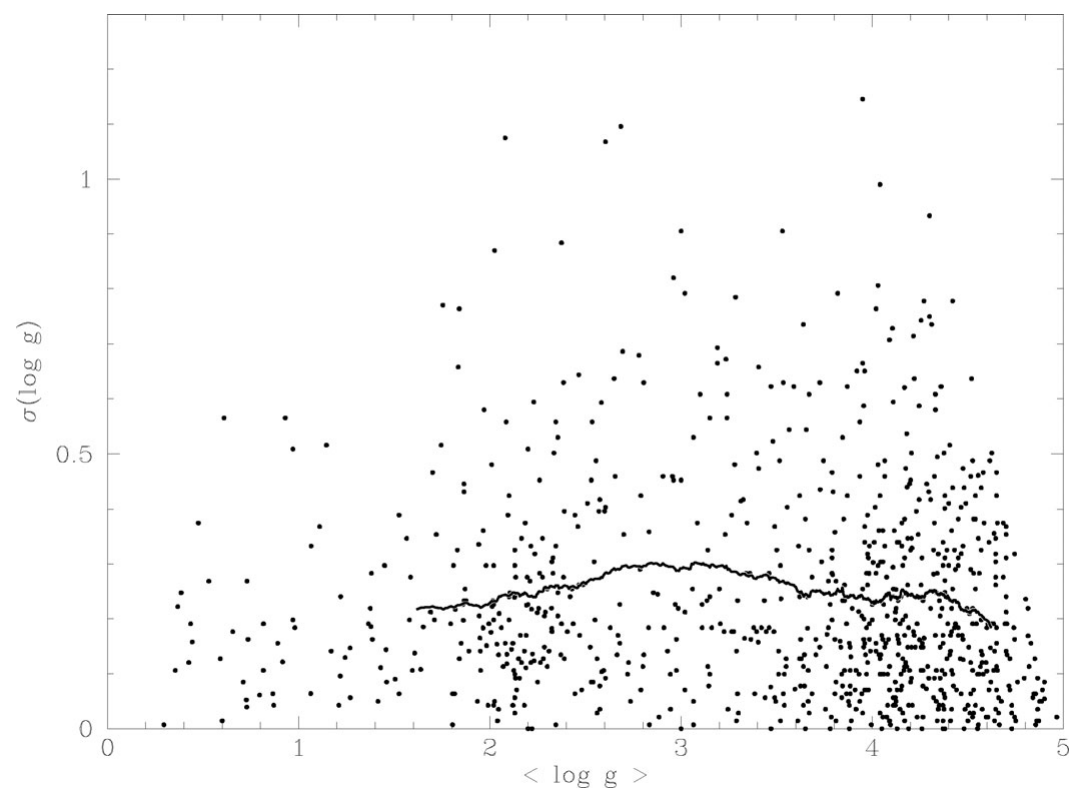

Figure 3. Accuracy of surface gravity as judged from repeated observations. Plot follows the style of Figure 2. 


\subsection{Ongoing Efforts}

RAVE's primary goal is to measure accurate radial velocities for stars in the Southern hemisphere. The quality of the RAVE spectra also enables us to measure atmospheric parameters $\left(\log g,[\mathrm{M} / \mathrm{H}]\right.$ and $\left.\mathrm{T}_{\text {eff }}\right)$ which can be used to select subsample of the catalogue with specific properties. For example, the combination of colours and $\log g$ measurements from RAVE permits an accurate selection of red clump giants (Veltz et al. 2008, Siebert et al. 2008). For this population, the narrow luminosity function enables us to obtain distances to $20 \%$ from the apparent magnitude alone.

However, the measurement of the atmospheric parameters is non-trivial at RAVE resolution and the transformation from the measured parameters to the true parameters relies on calibration data. The RAVE collaboration constantly acquires data for standard and pseudo-standards stars, using both the $6 \mathrm{dF}$ instrument and high-resolution spectra from other instruments, with the aim to refine our calibration.

Despite the medium resolution of the RAVE spectra, chemical abundances for about 12 elements can be measured with a good accuracy $(\sim 0.2$ dex $)$ in the high signal to noise spectra $(S / N>80)$. The RAVE collaboration puts a particular effort on measuring and validating these abundances. For this purpose, RAVE also acquires spectra for nearby stars for which accurate abundances have been measured using high-resolution spectroscopy. So far, abundances have been derived for more than 20,000 RAVE targets, and the resulting measurements will be published in a seperate catalogue. Furthermore, we plan to publish a list of fast rotating (and generally hot) stars where the rotational broadening can be detected at the resolving power of RAVE spectra.

The knowledge of the $6 \mathrm{D}$ phase space requires that we can transform the proper motions into spatial velocities. This step relies on good distance estimates for individual stars, which can be obtained from comparison of apparent and absolute magnitudes, the latter inferred from values of atmospheric parameters. The availability of distances in the near future will allow to exploit the full potential of the RAVE catalogue, permitting to

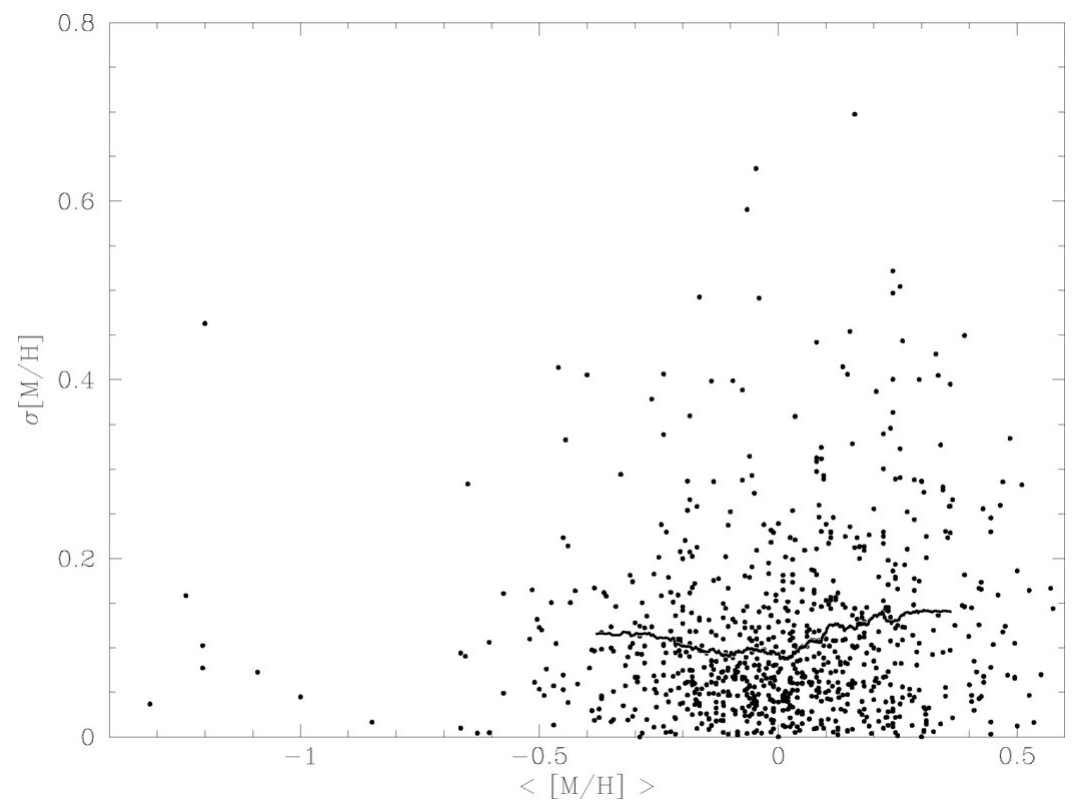

Figure 4. Accuracy of metallicity as judged from repeated observations. Plot follows the style of Figure 2. 
study the detailed shape of the phase space and reveal new details of the formation of the Solar neighbourhood.

\section{Conclusions}

The RAVE collaboration has released a second catalogue in July 2008, reporting radial velocities for 51,829 spectra and 49,327 different stars, randomly selected in the magnitude range of $9<I<12$ and located more than $25^{\circ}$ away from the Galactic plane. This release doubles the size of the previously published catalogue. The typical error of the published radial velocities is between 1.3 and $1.7 \mathrm{~km} \mathrm{~s}^{-1}$.

In addition to radial velocities, the catalogue contains, for the first time, atmospheric parameter measurements for more than 20,000 spectra. Uncertainties for a typical RAVE star are of the order of $400 \mathrm{~K}$ in temperature, of 0.5 dex in gravity, and of 0.2 dex in metallicity, but the error depends on the $\mathrm{S} / \mathrm{N}$ and can vary by a factor of $\sim 2$ for stars at the extreme ends of the $\mathrm{S} / \mathrm{N}$ range. Comparison of parameter measurements for repeated observations of the same targets indicates that these estimates are conservative and that the true errors may be smaller.

The survey continues to collect spectra. Currently more than 300,000 spectra have been collected and are currently being processed. Acquisition of new calibration data is also underway, enabling us to refine the atmospheric parameter measurements as well as radial velocities, and represents an import ongoing effort. Other current activities include the measurement of chemical abundances and distances which will be released as companion catalogues in the future.

The RAVE catalogue can be used to study the formation of the Milky Way and, for example, the collaboration succeeded in refining significantly the measurement of the

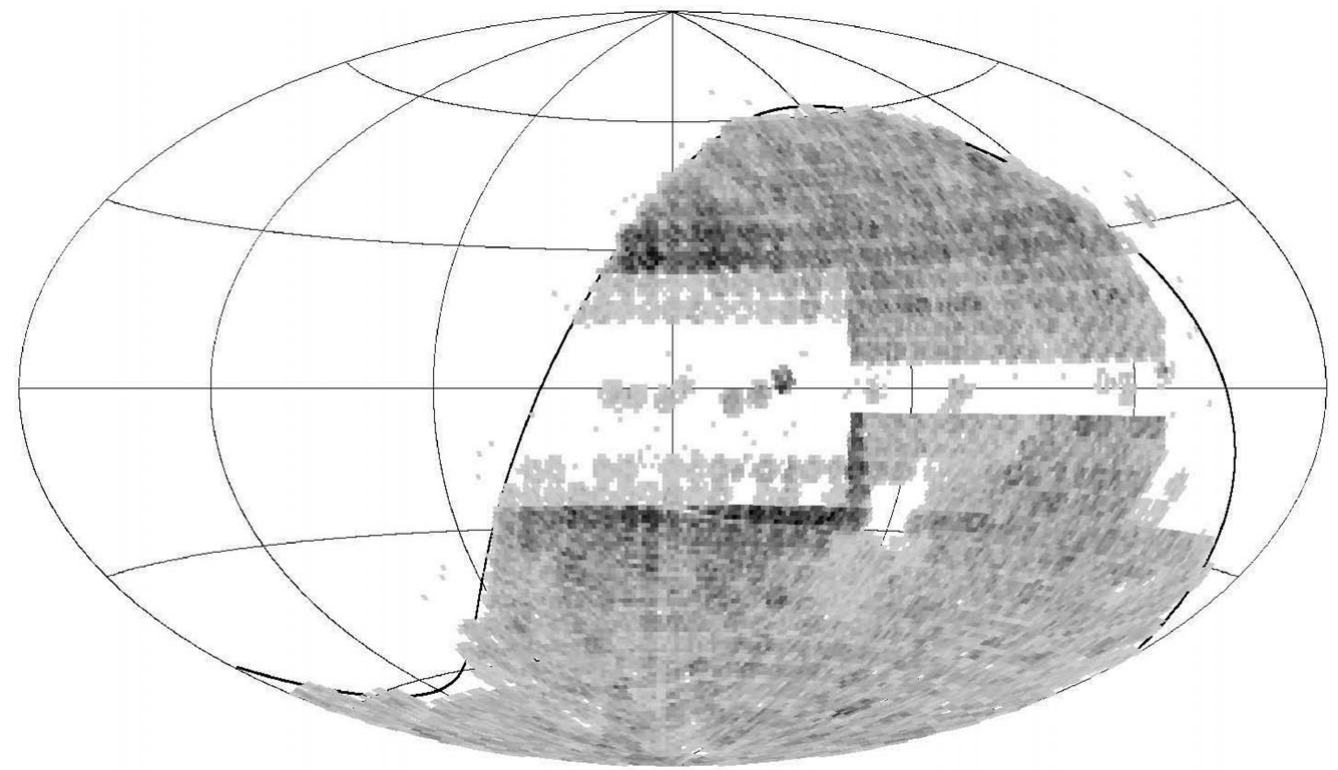

Figure 5. Aitoff projection in Galactic coordinates of the number of RAVE spectra per square degree. Only the spectra observed before 6th of July 2008 and which successfully passed the quality controls are included. They cover 16,730 square degrees of the Sourhern sky, with a mode of 8 spectra per square degree. Density is linearly coded in shades of grey from zero in white to the maximum of 93 spectra per square degree in black. Spectra on the Galactic plane were observed for calibration and test purposes. The S-shaped curve marks the celestial equator. 
local escape velocity using the high velocity stars found in the RAVE catalogue. Further studies carried out by the collaboration include the search for vertical stream of matter in the Solar neighborhood; a new determination of the vertical structure of the Galactic disc; the study of diffuse interstellar bands in the Galactic plane and their correlation to the interstellar extinction; the measurement of the inclination of the velocity ellipsoid towards the Galactic plane at $1 \mathrm{kpc}$ below the plane.

As the survey progresses and new data become available, the RAVE catalogue will enable us to refine our view on the structure and formation of the Milky Way, paving the way for new insights on the formation of galaxies.

\section{Acknowledgements}

Funding for RAVE has been provided by the Anglo-Australian Observatory, the Astrophysical Institute Potsdam, the Australian Research Council, the German Research foundation, the National Institute for Astrophysics at Padova, The Johns-Hopkins University, the Netherlands Research School for Astronomy, the Natural Sciences and Engineering Research Council of Canada, the Slovenian Research Agency, the Swiss National Science Foundation, the National Science Foundation of the USA (AST-0508996), the Netherlands Organisation for Scientific Research, the Particle Physics and Astronomy Research Council of the UK, Opticon, Strasbourg Observatory, and the Universities of Basel, Cambridge, and Groningen. The RAVE Web site is at www.rave-survey.org.

\section{References}

Munari, U. et al. 2008, Aछ A, 488, 969

Seabroke, G. M. et al. 2008, MNRAS, 384, 11

Siebert, A. et al. 2008, MNRAS, accepted (arXiv:0809.0615)

Smith, M. C. et al. 2007, MNRAS, 379, 755

Steinmetz, M. et al. 2006, AJ, 132, 1645

Veltz, L. et al. 2008, A\&A, 480, 753

Zwitter, T. et al. 2008, AJ, 136, 421 